\title{
Prediction of Acute COPD Exacerbation in the Swiss Multicenter COPD Cohort Study (TOPDOCS) by Clinical Parameters, Medication Use, and Immunological Biomarkers
}

\author{
Simona Tabea Huebner ${ }^{\mathrm{a}} \mathrm{b}$ Simona Henny ${ }^{\mathrm{a}}$ Stéphanie Giezendanner ${ }^{\mathrm{a}}$ Thomas Brack $^{\mathrm{c}}$ \\ Martin Brutsche ${ }^{d}$ Prashant Chhajed $^{\mathrm{a}}$ Christian Clarenbach $^{\mathrm{e}}$ Thomas Dieterle $^{\mathrm{b}}$ Adrian Egli ${ }^{\mathrm{f}, \mathrm{g}}$ \\ Martin Frey $^{\mathrm{h}}$ Ingmar Heijnen ${ }^{i}$ Sarosh Irani ${ }^{j}$ Noriane Andrina Sievi ${ }^{\mathrm{e}}$ Robert Thurnheer $^{\mathrm{k}}$ \\ Marten Trendelenburg ${ }^{b, l}$ Malcolm Kohler ${ }^{\mathrm{e}}$ Anne Barbara Leuppi-Taegtmeyer ${ }^{\mathrm{b}, \mathrm{m}}$ \\ Joerg Daniel Leuppi ${ }^{a, b}$ \\ aUniversity Clinic of Medicine, Cantonal Hospital Baselland, Liestal, Switzerland; ${ }^{b}$ Faculty of Medicine, University of \\ Basel, Basel, Switzerland; 'Department of Internal Medicine, Cantonal Hospital Glarus, Glarus, Switzerland; 'Division \\ of Respiratory Medicine, Cantonal Hospital St. Gallen, St. Gallen, Switzerland; 'Division of Respiratory Medicine, \\ University Hospital Zurich, Zurich, Switzerland; fDivision of Clinical Microbiology, University Hospital Basel, \\ University of Basel, Basel, Switzerland; ${ }^{9}$ Applied Microbiology Research, Department of Biomedicine, University of \\ Basel, Basel, Switzerland; ' $D i v i s i o n$ of Respiratory Medicine, Hospital Barmelweid, Barmelweid, Switzerland; 'Division \\ of Medical Immunology, Department of Laboratory Medicine, University Hospital Basel, Basel, Switzerland; jDivision \\ of Respiratory Medicine, Cantonal Hospital Aarau, Aarau, Switzerland; ${ }^{k}$ Division of Respiratory Medicine, Cantonal \\ Hospital Münsterlingen, Münsterlingen, Switzerland; 'Division of Internal Medicine, University Hospital Basel, and \\ Clinical Immunology, Department of Biomedicine, University of Basel, Basel, Switzerland; 'mepartment of Clinical \\ Pharmacology, University Hospital Basel, Basel, Switzerland
}

\section{Keywords}

IFN-lambda rs8099917 - lgG2 - Acute exacerbation of COPD . $\mathrm{FEV}_{1} \%$ predicted $\cdot$ COPD medication

\begin{abstract}
Background and Objective: Whether immunological biomarkers combined with clinical characteristics measured during an exacerbation-free period are predictive of acute exacerbation of chronic obstructive pulmonary disease (AECOPD) frequency and severity is unknown. Method: We measured immunological biomarkers and clinical characteristics in 271 stable chronic obstructive pulmonary disease (COPD) patients (67\% male, mean age 63 years) from "The
\end{abstract}

Obstructive Pulmonary Disease Outcomes Cohort of Switzerland" cohort on a single occasion. One-year follow-up data were available for 178 patients. Variables independently associated with AECOPD frequency and severity were identified by multivariable regression analyses. Receiver operating characteristic analysis was used to obtain optimal cutoff levels and measure the area under the curve (AUC) in order to assess if baseline data can be used to predict future AECOPD. Results: Higher number of COPD medications (adjusted incident rate ratio [aIRR] 1.17) and platelet count (aIRR

S.T.H. and S.H. contributed equally.

The TOPDOCS cohort study is registered at Clinical Trails.gov NCT01527773.
(C) 2021 The Author(s)

Published by S. Karger AG, Basel

This is an Open Access article licensed under the Creative Commons Attribution-NonCommercial-4.0 International License (CC BY-NC) (http://www.karger.com/Services/OpenAccessLicense), applicable to the online version of the article only. Usage and distribution for commercial purposes requires written permission.
Correspondence to:

Joerg Daniel Leuppi, joerg.leuppi@ @sbl.ch 
1.03), and lower $\mathrm{FEV}_{1} \%$ predicted (aIRR 0.84 ) and IgG2 (aIRR 0.84 ) were independently associated with AECOPD frequen$c y$ in the year before baseline. Optimal cutoff levels for experiencing frequent $(>1)$ AECOPD were $\geq 3$ COPD medications $(A \cup C=0.72), F E V_{1} \leq 40 \%$ predicted $(A \cup C=0.72)$, and $\lg G 2$ $\leq 2.6 \mathrm{~g} / \mathrm{L}$ (AUC = 0.64). The performance of a model using clinical and biomarker parameters to predict future, frequent AECOPD events in the same patients was fair (AUC = 0.78 ) but not superior to a model using only clinical parameters (AUC $=0.79$ ). The IFN-lambda rs8099917GG-genotype was more prevalent in patients who had severe AECOPD. Conclusions: Clinical and biomarker parameters assessed at a single point in time correlated with the frequency of $A E-$ COPD events during the year before and the year after assessment. However, only clinical parameters had fair discriminatory power in identifying patients likely to experience frequent AECOPD.

(c) 2021 The Author(s) Published by S. Karger AG, Basel

\section{Introduction}

Chronic obstructive pulmonary disease (COPD) is currently the third leading age-adjusted cause of death due to a noncommunicable disease worldwide [1]. Acute exacerbations of COPD (AECOPD) occur frequently [2] and are associated with disease progression, worsening health status, increased hospitalization rates, high healthcare costs, and significant mortality [3]. The ability to identify patients who are at increased risk of AECOPD can have a major impact on clinical management, quality of life, and healthcare costs. Although clinical characteristics, questionnaire scores, and medication use show significant associations with AECOPD [4-7], they include subjective values that depend on the accuracy of patients' reporting and physicians' recording, and in the case of the lung, function measurement on patient and physician effort. Therefore, we were interested to determine whether objective measurements such as immunological and genetic biomarkers in combination with clinical parameters can predict future AECOPD events.

Patients with frequent exacerbations have altered lower airway bacterial colonization and are more susceptible to viral infection because airway and systemic inflammation are increased $[8,9]$. The risk for infections depends on several immunological factors, making these potential candidate biomarkers in COPD patients. IgG deficiency, especially of IgG1 and IgG2 [10] and subnormal IgA levels [11], both affect AECOPD frequency and severity. Mannose-binding lectin (MBL), a recognition protein of the lectin pathway triggering complement activation, has also been described to influence innate immune responses in patients with AECOPD. MBL deficiency due to MBL2 gene codon 54 polymorphisms increases the risk of frequent and severe AECOPD [12]. Another immunological marker of interest is interferon lambda (IFNlambda), which is produced by dendritic and airway epithelial cells and is involved in both innate and adaptive immune responses $[13,14]$. IFN-lambda has direct antiviral properties; however, it may modulate the antibody response to viral infection [15]. A key feature of IFNlambda is its genetic variation determined by single-nucleotide polymorphisms (SNPs) in the IFN-lambda 3/4 gene region [16] such as the noncoding rs8099917 SNP which has previously been investigated in patients with viral infections [15, 17]. Furthermore, COPD patients with the rs8099917GG genotype are at increased risk for severe exacerbation (AECOPD requiring hospitalization) [18], possibly due to reduced direct antiviral activity of IFN-lambda. The combination of innate and humoral immune deficiencies may therefore be an important risk factor for AECOPD. Accordingly, we were interested to determine the association of clinical parameters and immunological biomarkers with AECOPD. We hypothesized that clinical parameters combined with immunological biomarkers can be used to predict future AECOPD.

\section{Methods}

Study Aims and Design

We investigated the association between clinical parameters and immunological biomarkers with the frequency and severity of past and future AECOPD. The study examined a subsample of patients from "The Obstructive Pulmonary Disease Outcomes Cohort of Switzerland" (TOPDOCS) cohort. TOPDOCS is a comprehensively characterized cohort of COPD patients living in Switzerland. The database was established to facilitate high-quality research on the pathogenesis, treatment, and clinical course of COPD [19]. In this prospective, noninterventional cohort study, patients with COPD were recruited from respiratory departments in 7 Swiss teaching hospitals. The primary outcome of this study was AECOPD frequency, the secondary outcome AECOPD severity. The primary endpoint measured was the area under the receiver operating characteristic curve of a model distinguishing between infrequent and frequent AECOPD. This was a hypothesisgenerating study.

The study was conducted in accordance with the Declaration of Helsinki of the World Medical Association and has been registered at Clinical Trails.gov NCT01527773. Ethics committees of all participating centers approved the study (lead center Zurich KEKZH-NR: 2011-0106).
Huebner et al. 


\section{Study Participants}

Patients aged between 40 and 75 years with mild to very severe COPD corresponding to Global Initiative for Chronic Obstructive Lung Disease (GOLD)-stages I-IV were included during an exacerbation-free interval of at least 6 weeks. Exclusion criteria were mental or physical restrictions impairing the ability to provide written informed consent or to participate in study visits. Written informed consent was obtained from all patients. For the current study, patients were included between October 2009 and December 2015.

\section{Baseline Assessment}

General information (age, sex, weight, and height), information about COPD (number of exacerbations and hospitalizations in the previous year), COPD medication, and symptoms were recorded, and vital parameters and lung function were measured at baseline. Exacerbations were defined as subject-reported worsening in respiratory health, requiring therapy with systemic corticosteroids and/or antibiotics. Frequent exacerbations were defined as 2 or more exacerbations in 1 year, and severe exacerbations were defined as those leading to hospitalization or emergency room visits [9]. Blood was taken for measuring immunological biomarkers including MBL and immunoglobulins (IgA, IgM, IgE, IgG, IgG1, IgG2, IgG3, and IgG4) and the genotype of INF-lambda rs8099917. Further details about the measurement of clinical parameters and biomarker analyses are given in the online supplementary material (see www.karger.com/doi/10.1159/000520196 for all online suppl. material). Study participants were contacted 1 year after study inclusion, and details of AECOPD in the past year were recorded.

\section{Statistical Methods}

Patient characteristics, clinical parameters, lung function parameters, and serum levels of biomarkers are given as mean and standard deviation, or median and interquartile range (IQR) if data were not normally distributed. Univariable tests were used to compare patients experiencing 0,1 , and $\geq 2$ AECOPD events or patients with and without a severe AECOPD event.

Incidence rate ratios (IRRs) for an AECOPD event were obtained by univariable Poisson regression, and odds ratios (OR) for frequent $(>1)$ or severe AECOPD events by univariable logistic regression. Adjusted IRRs for an AECOPD event were obtained using multivariable Poisson regression models in order to identify variables that were independently associated with the number of AECOPD events in the year before baseline (shown in online suppl. Fig. 1). In order to build a receiver operating characteristic (ROC) model, we dichotomized the outcome variable in infrequent ( 0 or 1$)$ and frequent $(>1)$ AECOPD. Multivariable logistic regression was used to obtain adjusted odds ratios for frequent AECOPD events (shown in online suppl. Fig. 1) and for the occurrence of severe AECOPD (AECOPD requiring hospitalization) during the year before baseline (shown in online suppl. Fig. 2). We used the model estimates to predict frequent or infrequent $\mathrm{AE}$ COPD events for the year after baseline, and compared the predicted and observed outcomes for each case. To evaluate the performance of the model, ROC curves were plotted. Furthermore, we built multivariable models with clinical and/or biomarker predictor variables and plotted them by ROC-curve in order to compare their performance in predicting frequent AECOPD before or after baseline. We determined optimal cutoff values for each variable

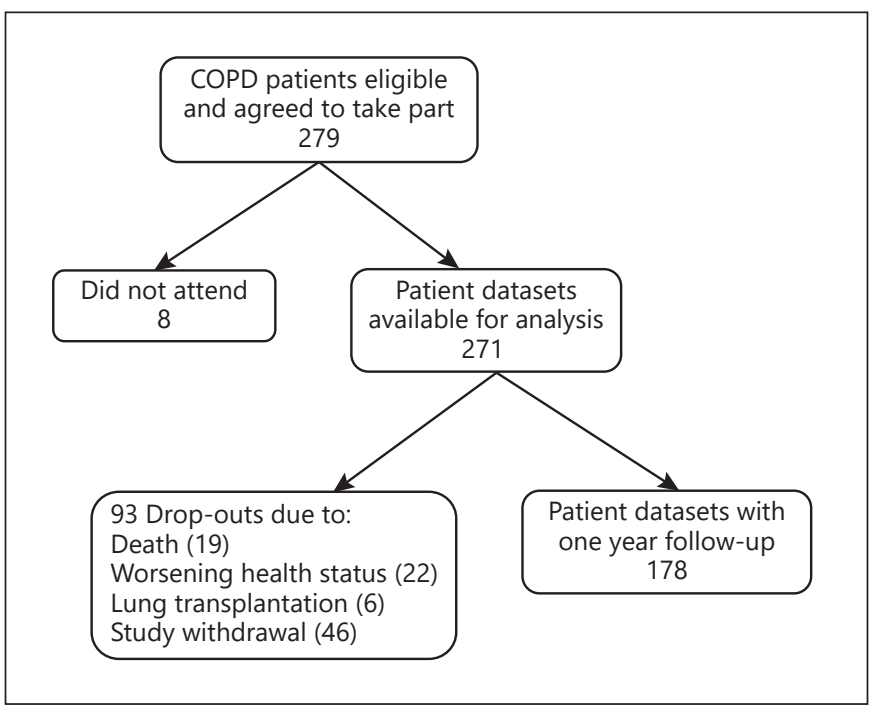

Fig. 1. Study flowchart showing the number of patient datasets included in the analysis of AECOPD events during the year before and after study inclusion. Online supplementary Figures 1 and 2 show further details regarding the analyses. AECOPD, acute exacerbation of chronic obstructive pulmonary disease; COPD, chronic obstructive pulmonary disease.

included in the multivariable model for distinguishing patients with infrequent ( 0 or 1$)$ and frequent $(>1)$ AECOPD events during the year before baseline using univariable ROC analysis. Sensitivity, specificity, and positive and negative predictive values were calculated by contingency tables. The discriminatory power was indicated by the area under the curve (AUC), whereby $0.5-0.6$ was considered fail, 0.6-0.7 poor, 0.7-0.8 fair, 0.8-0.9 good, and 0.91.0 very good [20].

In order to account for possible confounding due to correlations within individuals, we determined the association of previous AECOPD events with AECOPD during the year after baseline using Spearman's rank correlation coefficient. To further evaluate for a potential effect of previous AECOPD on future AECOPD, we built 2 multivariable logistic regression models. The first model accounted for the effect of variables which were significantly associated with frequent AECOPD in the previous year on frequent AECOPD during the year after baseline. The second model included the variables of the first model as well as the number of AECOPD events in the previous year. The discriminatory power (AUC) of these 2 models for distinguishing between infrequent ( 0 or 1) or frequent (>1) AECOPD during follow-up was then assessed by multivariable ROC curve analysis.

Analyses were conducted using $\mathrm{R}$ statistical software version 3.1.3 (RStudio: Integrated Development for R. RStudio, PBC, Boston, MA, USA), and a $p$ value $<0.05$ was considered statistically significant. Further details are given in the online supplementary material. 
Table 1. Baseline characteristics and biomarkers of all patients $(n=271)$ and of the subgroup with follow-up at year $1(n=178)$

\begin{tabular}{|c|c|c|c|c|}
\hline Parameter & All patients & $n$ & $\begin{array}{l}\text { Patients with } 1 \\
\text { year follow-up }\end{array}$ & $n$ \\
\hline Male, $n(\%)$ & $182(67)$ & & $127(71)$ & \\
\hline Age, years & $64( \pm 7.6)$ & & $64( \pm 7.1)$ & \\
\hline $\mathrm{BMI}, \mathrm{kg} / \mathrm{m}^{2}$ & $26(23-29)$ & & $26(23-28)$ & \\
\hline Current smokers, $n(\%)$ & $66(24)$ & & $44(25)$ & \\
\hline Pack years of smoking & $40(30-60)$ & 270 & $40(30-60)$ & 177 \\
\hline Systolic blood pressure, $\mathrm{mm} \mathrm{Hg}$ & $131( \pm 17)$ & 269 & $133( \pm 18)$ & 176 \\
\hline Diastolic blood pressure, $\mathrm{mm} \mathrm{Hg}$ & $80( \pm 11)$ & 269 & $81( \pm 10)$ & 176 \\
\hline Heart rate, beats per minute & $79( \pm 14)$ & 267 & $78( \pm 13)$ & 175 \\
\hline Oxygen saturation breathing room air, $\%$ & $95(93-96)$ & 259 & 95 (93-96) & 163 \\
\hline $\mathrm{FEV}_{1} / \mathrm{FVC}, \%$ & $46(35-60)$ & & $47(37-59)$ & \\
\hline $\mathrm{FEV}_{1} \%$ predicted, $\%$ & $44(31-65)$ & & $46(33-65)$ & \\
\hline CAT-score & $16( \pm 7)$ & 267 & $15( \pm 7)$ & 177 \\
\hline SF-6D V1 & $0.69(0.61-0.80)$ & 263 & $0.71(0.62-0.81)$ & 175 \\
\hline SF-6D V2 & $0.68(0.59-0.80)$ & 263 & $0.71(0.60-0.81)$ & 175 \\
\hline GOLD stage & $3(2-3)$ & & $3(2-3)$ & \\
\hline $1, n(\%)$ & $24(9)$ & & $16(9)$ & \\
\hline $2, n(\%)$ & $88(32)$ & & $64(36)$ & \\
\hline $3, n(\%)$ & $100(37)$ & & $68(38)$ & \\
\hline $4, n(\%)$ & $59(22)$ & & $30(17)$ & \\
\hline MMRC score & $2(1-3)$ & 270 & $1(1-2)$ & \\
\hline \multicolumn{5}{|c|}{ Patients experiencing $0,1,2,3,4$, and $\geq 5$ AECOPD events per year, $n(\%)$} \\
\hline 0 & $131(48)$ & & $110(61)$ & \\
\hline 1 & $74(27)$ & & $44(25)$ & \\
\hline 2 & $36(13)$ & & $18(10)$ & \\
\hline 3 & $16(6)$ & & $4(2)$ & \\
\hline 4 & $5(2)$ & & $1(1)$ & \\
\hline 5 or more & $8(3)$ & & $1(1)$ & \\
\hline \multicolumn{5}{|c|}{ Patients and number of COPD medication, $n(\%)$} \\
\hline 0 & $40(15)$ & & $26(15)$ & \\
\hline 1 & $25(9)$ & & $19(10)$ & \\
\hline 2 & $43(16)$ & & $31(17)$ & \\
\hline 3 & $99(37)$ & & $67(38)$ & \\
\hline 4 & $42(15)$ & & $24(13)$ & \\
\hline 5 & $14(5)$ & & $7(4)$ & \\
\hline 6 & $6(2)$ & & $3(2)$ & \\
\hline 7 & $2(1)$ & & $1(1)$ & \\
\hline \multicolumn{5}{|c|}{ Patients and type of maintenance inhaled therapy, $n(\%)$} \\
\hline LAMA & $19(7)$ & & $14(8)$ & \\
\hline LABA & $8(3)$ & & $4(2)$ & \\
\hline LAMA + LABA & $17(6)$ & & $13(7)$ & \\
\hline LAMA + ICS & $6(2)$ & & $5(3)$ & \\
\hline$L A B A+I C S$ & $38(14)$ & & $24(13)$ & \\
\hline$L A M A+L A B A+I C S$ & $140(52)$ & & $89(50)$ & \\
\hline \multicolumn{5}{|l|}{ As required inhaled medication } \\
\hline SABA & $72(27)$ & & $37(21)$ & \\
\hline SAMA & $30(11)$ & & $18(10)$ & \\
\hline \multicolumn{5}{|l|}{ Add-on therapies } \\
\hline Oral steroids & $11(4)$ & & $5(3)$ & \\
\hline Theophyllin & $10(4)$ & & $6(3)$ & \\
\hline Roflumilast & $4(1)$ & & $3(2)$ & \\
\hline NTproBNP, ng/L & $84(39-149)$ & 149 & $77(40-150)$ & 78 \\
\hline $\mathrm{CRP}, \mathrm{mg} / \mathrm{L}$ & $2.2(1.0-4.8)$ & 160 & $2.0(1.0-3.7)$ & 86 \\
\hline White blood cell count, $\times 10^{9} / \mathrm{L}$ & $7.7(6.3-9.1)$ & 269 & $7.6(6.2-8.9)$ & 176 \\
\hline Platelet count, $\times 10^{9} / \mathrm{L}$ & $263( \pm 72)$ & 269 & $262( \pm 72)$ & 176 \\
\hline
\end{tabular}


Table 1 (continued)

\begin{tabular}{|c|c|c|c|c|}
\hline Parameter & All patients & $n$ & $\begin{array}{l}\text { Patients with } 1 \\
\text { year follow-up }\end{array}$ & $n$ \\
\hline Creatinine, $\mu \mathrm{mol} / \mathrm{L}$ & $81( \pm 26)$ & 161 & $82( \pm 29)$ & 86 \\
\hline Bilirubin, $\mu \mathrm{mol} / \mathrm{L}$ & $7(5-10)$ & 146 & $7(5-10)$ & 79 \\
\hline $\mathrm{MBL}, \mu \mathrm{g} / \mathrm{mL}$ & $1.4(0.4-2.8)$ & 270 & $1.5(0.4-2.8)$ & 177 \\
\hline$<0.1, n(\%)$ & $40(15)$ & & $26(15)$ & \\
\hline$<0.5, n(\%)$ & $82(30)$ & & $52(29)$ & \\
\hline $\lg A, g / L$ & $2.2(1.6-3.1)$ & 269 & $2.3(1.7-3.2)$ & 176 \\
\hline $\lg M, g / L$ & $0.8(0.5-1.3)$ & 269 & $0.8(0.5-1.2)$ & 176 \\
\hline $\mathrm{lgE}, \mathrm{IU} / \mathrm{mL}$ & $49(21-130)$ & 246 & $51(21-142)$ & 162 \\
\hline $\operatorname{lgG}, g / L$ & $9.2( \pm 2.7)$ & 270 & $9.3( \pm 2.3)$ & 177 \\
\hline $\operatorname{lgG} 1, \mathrm{~g} / \mathrm{L}$ & $6.0( \pm 2)$ & 269 & $6.1( \pm 1.6)$ & 176 \\
\hline $\lg G 2, g / L$ & $2.6(1.8-3.5)$ & 269 & $2.7(1.9-3.6)$ & 176 \\
\hline $\operatorname{lgG} 3, g / L$ & $0.4(0.2-0.5)$ & 269 & $0.4(0.2-0.5)$ & 176 \\
\hline $\operatorname{lgG} 4, \mathrm{~g} / \mathrm{L}$ & $0.4(0.2-0.7)$ & 269 & $0.4(0.2-0.7)$ & 176 \\
\hline rs8099917 & & 181 & & 110 \\
\hline Patients with TT genotype, $n(\%)$ & $79(44)$ & & $53(48)$ & \\
\hline Patients with TG genotype, $n(\%)$ & $98(54)$ & & $56(51)$ & \\
\hline Patients with GG genotype, $n(\%)$ & $4(2)$ & & $1(1)$ & \\
\hline
\end{tabular}

Values are expressed as mean ( \pm standard deviation) or median (interquartile range), unless otherwise stated. If data were not available from all patients, $n$ gives the number for whom data were available. $M B L$, mannosebinding lectin; CRP, C-reactive protein; SAMA, short-acting muscarinic antagonists; SABA, short-acting beta agonists; LAMA, long-acting muscarinic antagonist; LABA, long-acting beta agonist; ICS, inhaled corticosteroid; COPD, chronic obstructive pulmonary disease; AECOPD, acute exacerbation of chronic obstructive pulmonary disease; GOLD, Global Initiative for Chronic Obstructive Lung Disease.

\section{Results}

\section{Study Participants}

Two hundred seventy-one of the 279 COPD patients who agreed to take part in the TOPDOC study attended for assessment (shown in Fig. 1). Baseline characteristics of all patients, and of the subgroup of 178 patients with 1 year follow-up, are given in Table 1. The mean age was 64 years, $67 \%$ were male, and the median GOLD stage was 3. The number of AECOPD events ranged from 0 to 11 per year (median 1, IQR 0-1 in the year before baseline; and median 0, IQR 0-1 in the year after baseline). 140 patients (52\%) experienced one or more exacerbations during the year prior to baseline. Of these, 57 (41\%, or $21 \%$ of all patients) were hospitalized at least once. $231 \mathrm{pa}-$ tients $(85 \%)$ were treated with inhaled medication at baseline (median 3 different inhaled medications, IQR $2-3)$. Of those, 140 (52\%) were treated with combination therapy consisting of a long-acting muscarinic antagonist (LAMA), a long-acting beta agonist (LABA), and an inhaled corticosteroid (ICS). As-needed short-acting muscarinic antagonists were prescribed to 30 patients (11\%) and short-acting beta agonists to 72 patients (27\%).

\section{Comparison between Patients with 0, 1, and $>1$}

\section{AECOPD Events}

Patients were grouped by how many AECOPD events $(0,1$, or $>1)$ they experienced in the year before or the year after baseline assessment. Patients who had experienced AECOPD events during the previous year had significantly higher pack years of smoking, heart rate, CATscores, GOLD stages, MMRC scores, number of COPD medications, and platelet counts, and lower body mass index (BMI), FEV1\% predicted, SF-6D V1 and V2, bilirubin, and serum IgM, IgE, and IgG (in particular IgG2) values than those who did not (Table 2). In the $178 \mathrm{pa}$ tients followed up after 1 year, patients with more frequent AECOPD had higher baseline values for heart rate, GOLD stage, MMRC score and number of COPD medication, and lower oxygen saturation and $\mathrm{FEV}_{1} \%$ predicted (Table 2). COPD medication type according to frequency of AECOPD and variables which did not differ significantly between groups are shown in online supplementary Table 1. Patients treated with LAMA/LABA/ICS at baseline were more likely to have experienced AECO$\mathrm{PD}$ during the past year and more likely to experience it in the following year (online suppl. Table 1). Further- 
Table 2. Baseline parameters according to frequency of AECOPD in the year before (first line, $n=271$ ) and after (second line, $n=178$ ) baseline

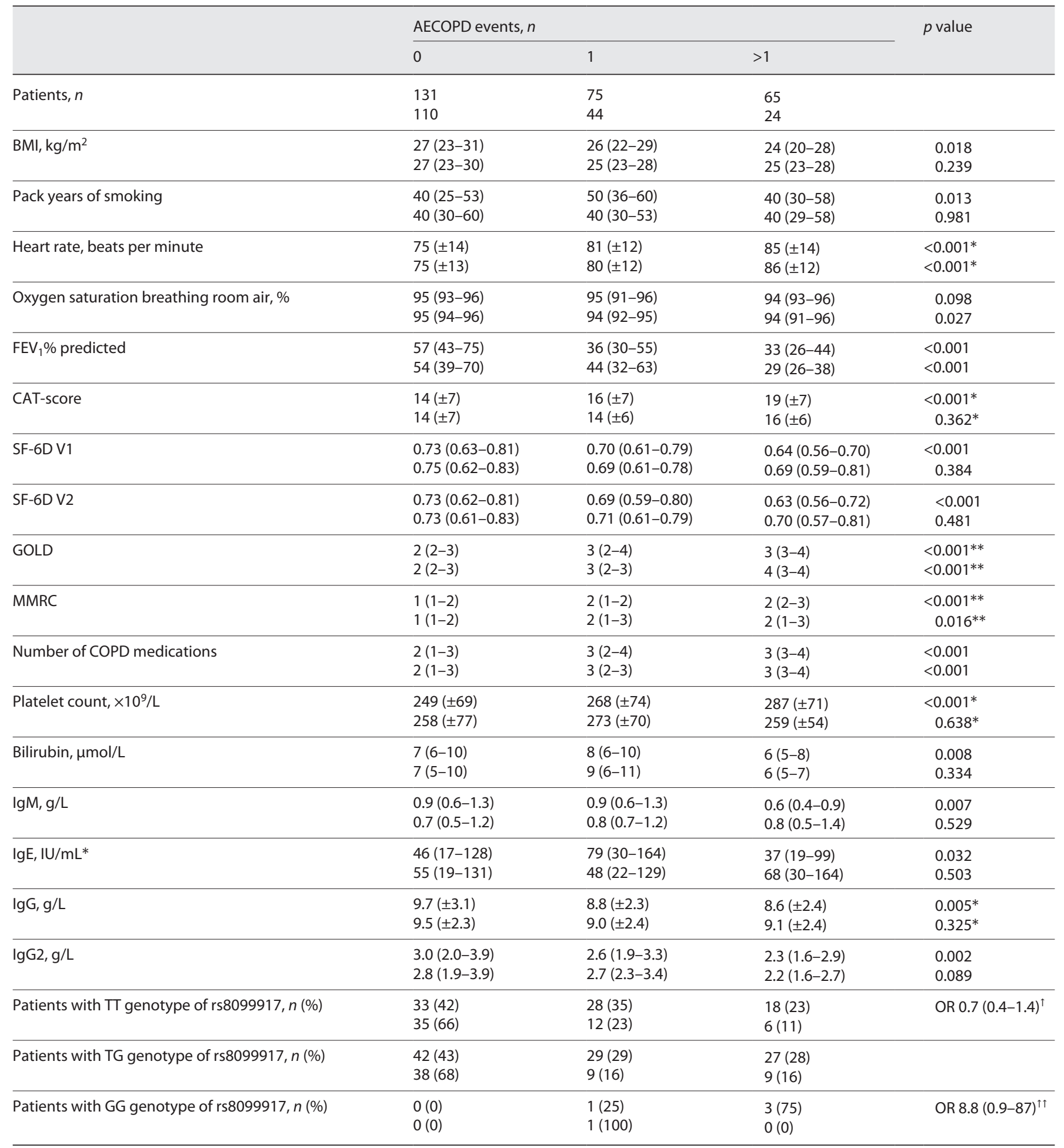

Unless otherwise stated, mean ( \pm standard deviation) or median (interquartile range) values are given. $p$ refers to the overall comparison between patients with 0 , 1, or >1 AECOPD. AECOPD, acute exacerbation of chronic obstructive pulmonary disease; GOLD, Global Initiative for Chronic Obstructive Lung Disease; COPD, chronic obstructive pulmonary disease; BMI, body mass index; OR, odds ratio. Kruskal-Wallis or * ANOVA, ${ }^{* *} \mathrm{X}^{2}$-test, ${ }^{* * *}$ Fisher's test, ${ }^{\dagger}$ OR and 95\% confidence interval for $>1$ exacerbations $T T$ versus TG/GG. ${ }^{\uparrow \uparrow}$ Odds ratio and $95 \%$ confidence interval for $>1$ exacerbations GG versus $T$ T/TG. 
Table 3. Univariable analyses of baseline parameters according to number of severe AECOPD events (AECOPD requiring emergency department visit or hospitalization) in the year before (first line, $n=271$ ) and after (second line, $n=178$ ) baseline

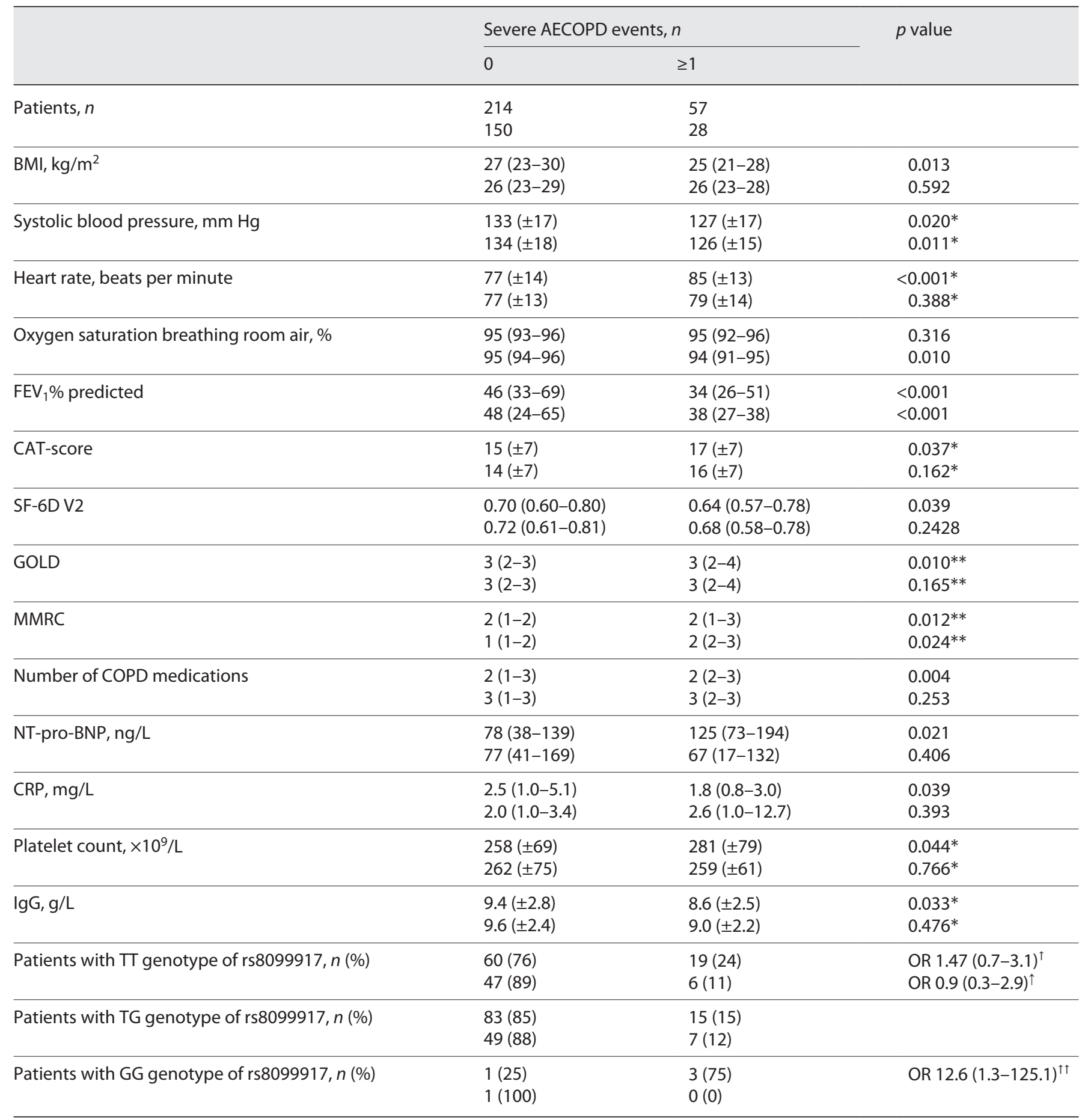

Unless otherwise stated, mean ( \pm standard deviation) or median (interquartile range) values are given. $p$ refers to the overall comparison between patients with and without severe AECOPD. OR, odds ratio; COPD, chronic obstructive pulmonary disease; GOLD, Global Initiative for Chronic Obstructive Lung Disease; AECOPD, acute exacerbation of chronic obstructive pulmonary disease; BMI, body mass index; COPD, chronic obstructive pulmonary disease. Mann-Whitney-U-Test or * Student's $t$ test, ${ }^{* *} x^{2}$-test, $* * *$ Fisher's test. ${ }^{\uparrow}$ OR and $95 \%$ confidence interval for hospitalization TT versus TG/GG. ${ }^{\uparrow}$ Odds ratio and 95\% confidence interval for hospitalization GG versus $\Pi$ T/TG. 
Table 4. Multivariable Poisson model for number of AECOPD events in the year before baseline assessment $(n=$ 255)

\begin{tabular}{|c|c|c|c|c|c|}
\hline \multirow[t]{2}{*}{ Parameter } & \multirow[t]{2}{*}{ AIRR } & \multicolumn{2}{|c|}{$95 \%$ confidence interval } & \multirow[t]{2}{*}{$p$ value } & \multirow{2}{*}{$\begin{array}{l}\text { Variance } \\
\text { inflation } \\
\text { factor }\end{array}$} \\
\hline & & lower & upper & & \\
\hline Sex (men vs. women) & 0.89 & 0.63 & 1.27 & 0.430 & 1.32 \\
\hline Age, per 10 years & 1.08 & 0.87 & 1.34 & 0.414 & 1.11 \\
\hline $\mathrm{BMI}$, per $5 \mathrm{~kg} / \mathrm{m}^{2}$ & 1.05 & 0.93 & 1.20 & 0.403 & 1.33 \\
\hline Current smokers versus nonsmoker & 0.95 & 0.64 & 1.41 & 0.793 & 1.23 \\
\hline Heart rate, per 10 beats per minute & 1.08 & 0.96 & 1.22 & 0.116 & 1.35 \\
\hline $\mathrm{FEV}_{1} \%$ predicted, per $10 \%$ & 0.84 & 0.76 & 0.93 & 0.0002 & 1.51 \\
\hline CAT-score & 1.02 & 0.99 & 1.06 & 0.058 & 1.84 \\
\hline SF-6D V1, per 0.1 units increase & 0.89 & 0.77 & 1.02 & 0.077 & 1.73 \\
\hline Number of COPD medications & 1.17 & 1.06 & 1.30 & 0.004 & 1.58 \\
\hline White blood cell count, per 1,000 cells/ $\mu \mathrm{L}$ & 0.99 & 0.93 & 1.05 & 0.605 & 1.50 \\
\hline Platelet count, per 10,000 platelets $/ \mu \mathrm{L}$ & 1.03 & 1.01 & 1.05 & 0.010 & 1.44 \\
\hline $\operatorname{lgG} 2$, per g/L & 0.84 & 0.74 & 0.95 & 0.006 & 1.25 \\
\hline
\end{tabular}

AECOPD, acute exacerbation of chronic obstructive pulmonary disease; COPD, chronic obstructive pulmonary disease; AIRR, adjusted incident rate ratio.

more, patients treated with LABA alone or in combination had a significantly higher mean heart rate $(80 \pm 14$ beats per minute with LABA vs. $74 \pm 12$ beats per minute without LABA, $p=0.001)$. Patients treated with ICS had significantly lower IgG2 levels ( $2.7 \pm 1.3$ vs. $3.1 \pm 1.7, p=$ $0.04)$ and a trend toward lower IgG levels ( $8.9 \pm 2.3$ vs. 9.7 $\pm 3.4, p=0.07)$. Patients with ICS therapy had a significantly higher GOLD stage ( 3 vs. $2, p<0.001$ ) and MMRC score ( 2 vs. $1, p<0.001$ ). There was a trend toward a higher likelihood of having experienced 2 or more AECOPD events during the previous year in patients with the rs8099917GG genotype (Table 2).

\section{Risk Factors for Severe AECOPD}

Patients who had experienced severe AECOPD during the year prior to baseline had significantly higher heart rates, CAT-scores, GOLD stages, MMRC scores, number of COPD medications, $\mathrm{N}$-terminal pro-B-type natriuretic peptide (NT-pro-BNP) and platelet counts, and a lower BMI, systolic blood pressure, $\mathrm{FEV}_{1} \%$ predicted, $\mathrm{SF}-6 \mathrm{D}$ V2, C-reactive protein, and serum IgG (Table 3). COPD medication type according to severity of AECOPD and variables which did not differ significantly between groups are shown in online supplementary Table 2. Patients who had previous severe AECOPD were more often treated with combined LAMA/LABA/ICS medication at baseline. Furthermore, patients with the rs8099917GG-genotype had more severe AECOPD during the previous year, with 3 of the 4 GG-genotype pa- tients requiring hospitalization. Patients with severe AECOPD events during the first follow-up year had significantly higher MMRC scores and lower systolic blood pressure, oxygen saturations, and $\mathrm{FEV}_{1} \%$ predicted at baseline compared to those without severe AECOPD events (Table 3). A subgroup analysis of 19 patients who died during the follow-up year showed that older age (67 \pm 7 in non-survivors vs. $62 \pm 8$ years in survivors, $p=$ 0.02 ), lower oxygen saturation ( $92 \pm 4$ vs. $94 \pm 3 \%$, $p=$ $0.04)$ and higher NT-pro-BNP (402 \pm 684 vs. $229 \pm 910$ $\mathrm{ng} / \mathrm{L}, p=0.005)$ were significantly associated with poor survival. The immunological markers IgG2 (non survivors: $3 \pm 1.4 \mathrm{~g} / \mathrm{L}$ vs. survivors: $2.8 \pm 2 \mathrm{~g} / \mathrm{L}, p=0.87$ ) and platelet count (non survivors: $234 \pm 64 \mathrm{G} / \mathrm{L}$ vs. survivors: $265 \pm 72 \mathrm{G} / \mathrm{L}, p=0.06$ ) were not significantly different between the 2 groups, although platelet count showed a trend to lower values among non-survivors.

\section{Factors Independently Associated with Exacerbation}

Frequency and Severity

Parameters significantly associated with the frequency of AECOPD events (univariable Poisson analysis, online suppl. Table 3) were included in a multivariable Poisson model, and adjusted IRRs for the occurrence of an AECOPD event during the year before baseline were determined (Table 4). The number of COPD medications and platelet count, together with lower $\mathrm{FEV}_{1} \%$ predicted and IgG2 levels, remained independently associated with AECOPD frequency. Parameters that were significantly as- 
Table 5. Multivariable logistic regression model of the association between baseline variables (clinical and biomarker variables combined) and patients with infrequent ( 0 or 1$)$ or frequent $(>1)$ AECOPD events in the year before baseline $(n=256)$

\begin{tabular}{|c|c|c|c|c|c|}
\hline \multirow[t]{2}{*}{ Parameter } & \multirow[t]{2}{*}{ AOR } & \multicolumn{2}{|c|}{ 95\% confidence interval } & \multirow[t]{2}{*}{$p$ value } & \multirow{2}{*}{$\begin{array}{l}\text { Variance } \\
\text { inflation factor }\end{array}$} \\
\hline & & lower & upper & & \\
\hline Sex (men vs. women) & 0.95 & 0.45 & 2.03 & 0.898 & 1.25 \\
\hline Age, per 10 years & 0.86 & 0.54 & 1.36 & 0.503 & 1.08 \\
\hline $\mathrm{BMI}$, per $5 \mathrm{~kg} / \mathrm{m}^{2}$ & 1.02 & 0.74 & 1.39 & 0.891 & 1.31 \\
\hline Heart rate, per 10 beats per minute & 1.30 & 1.00 & 1.69 & 0.052 & 1.16 \\
\hline $\mathrm{FEV}_{1}(\%)$ predicted, per $10 \%$ & 0.79 & 0.63 & 0.99 & 0.045 & 1.58 \\
\hline CAT-score & 1.05 & 0.99 & 1.12 & 0.128 & 1.75 \\
\hline SF-6D V1, per 0.1 units increase & 0.76 & 0.53 & 1.08 & 0.122 & 1.64 \\
\hline MMRC stage & 0.88 & 0.56 & 1.36 & 0.556 & 1.85 \\
\hline Number of COPD medications & 1.31 & 0.99 & 1.75 & 0.063 & 1.33 \\
\hline Platelet count, per 10,000 platelets $/ \mu \mathrm{L}$ & 1.05 & 0.99 & 1.10 & 0.058 & 1.14 \\
\hline $\lg G 2$, per $g / L$ & 0.73 & 0.52 & 0.99 & 0.061 & 1.20 \\
\hline
\end{tabular}

BMI, body mass index; COPD, chronic obstructive pulmonary disease; aOR, adjusted odds ratio; AECOPD, acute exacerbation of chronic obstructive pulmonary disease.

sociated with hospitalization or emergency room attendance for AECOPD (severe AECOPD-online suppl. Table 4) were included in a multivariable logistic model (online suppl. Table 5). Higher heart rate was independently associated with severe AECOPD in the year before baseline. Due to the correlations between GOLD stage and $\mathrm{FEV}_{1} \%$ predicted $(r=-0.99, p=0.001)$, SF-6D V1 and V2 $(r=0.99, p=0.001)$, and IgG with $\operatorname{IgG1}(r=0.85$, $p=0.02)$, and IgG2 $(r=0.70, p=0.04)$, we only included the second-mentioned parameter of each pair into the respective multivariate analyses.

\section{Using Baseline Data to Predict Frequent AECOPD}

Events during Follow-Up

Variables that were significantly associated with frequent $(>1)$ AECOPD events in the year before study inclusion (univariable logistic regression analysis, online suppl. Table 6) were further analyzed by multivariable logistic regression. In order to compare the contribution of clinical and biomarker variables to the multivariable model, we built 2 separate multivariable models (online suppl. Tables 7,8 ) and one model with clinical and biomarker variables combined (Table 5). In the combined model, only $\mathrm{FEV}_{1} \%$ predicted remained independently significant (Table 5). The multivariable ROC curve of the model including only clinical variables showed good discriminatory power for distinguishing between frequent and infrequent AECOPD during the year before baseline (shown in Fig. 2a, blue line, AUC 0.8), and fair discrimi- natory power for the follow-up year (shown in Fig. 2a, green line, AUC 0.79). The discriminatory power for distinguishing between frequent and infrequent AECOPD using biomarker variables alone was fair for the year before baseline, but poor for the follow-up year (AUC 0.7 and 0.6, respectively, shown in Fig. 2b). Combining biomarker and clinical variables only improved the discriminatory power from 0.8 to 0.81 for the year before baseline; there was no improvement in the model's performance for the year after baseline ( 0.79 vs. 0.78 , shown in Fig. 2a, c).

The cutoff values obtained by univariable ROC-analyses along with their corresponding sensitivity, specificity, and AUC values for distinguishing between patients who had had frequent $(>1)$ versus infrequent ( 0 or 1$)$ AECO$\mathrm{PD}$ events in the year before baseline are shown in $\mathrm{Ta}$ ble $6 . \mathrm{FEV}_{1} \%$ predicted and number of COPD medication showed fair AUC values for distinguishing between infrequent and frequent previous AECOPD in univariable ROC-analyses (Table 6, AUC both 0.72). Using the optimal-cutoff points, the positive and negative predictive values for FEV $1 \%$ predicted were $39 \%$ and $87 \%$ for having frequent AECOPD in the year before, and 27\% and 95\% in the year after baseline, respectively. For number of COPD medication, these figures were $35 \%$ and $93 \%$ in the year before, and $19 \%$ and $93 \%$ in the year after baseline, respectively.

The correlation between the number of AECOPD events in the year before and the year after baseline was 


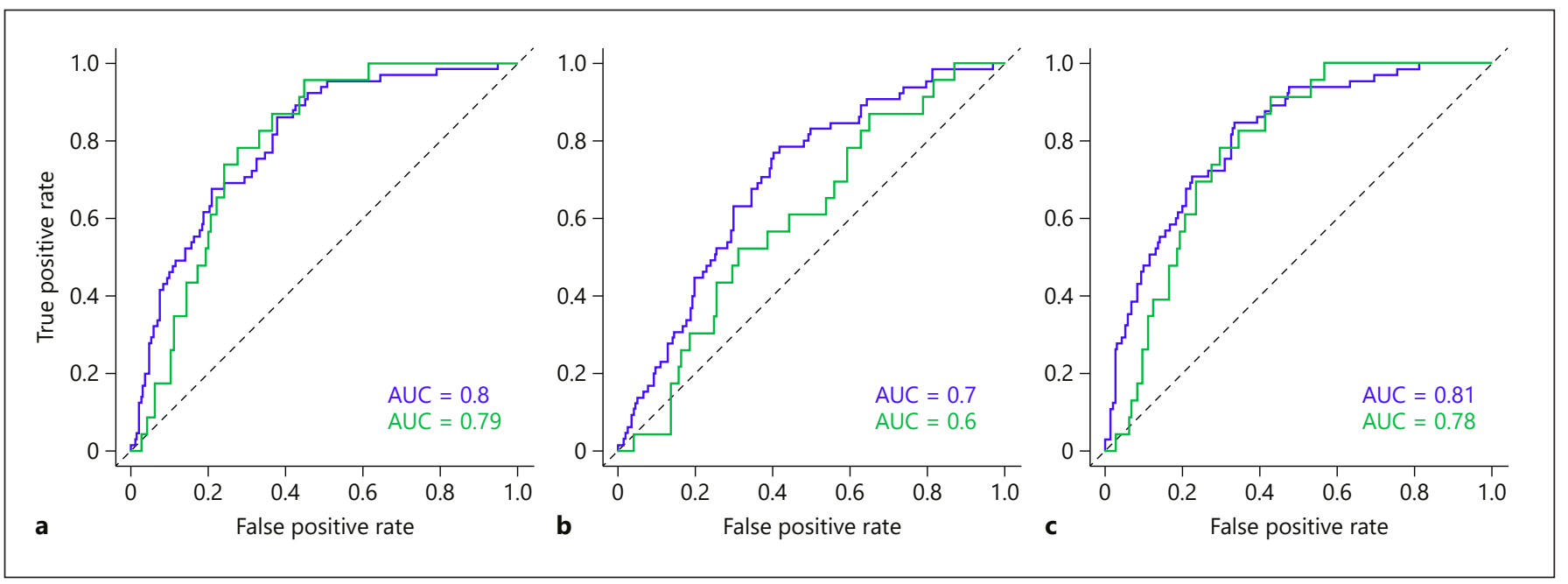

Fig. 2. Multivariable ROC curves with corresponding AUC values for discriminating between 0 or 1 and $>1 \mathrm{AE}$ COPD events in the year before (blue) and after (green) baseline. (a) Multivariable ROC curves using clinical parameters, (b) multivariable ROC curve using biomarker variables, and (c) multivariable ROC curve of both clinical and biomarker variables, combined. -----: line of no discrimination. ROC, receiver operating characteristic; AUC, area under the curve; AECOPD, acute exacerbation of chronic obstructive pulmonary disease.

Table 6. Optimal cutoff points obtained by ROC curve analyses with corresponding sensitivity and specificity for frequent $(>1)$ versus infrequent ( 0 or 1 ) AECOPD events in the year before baseline

\begin{tabular}{lllll}
\hline Parameter & Cutoff & Sensitivity, $\%$ & Specificity, \% & AUC (95\% confidence interval) \\
\hline BMI & $\leq 25 \mathrm{~kg} / \mathrm{m}^{2}$ & 58 & 60 & $0.61(0.52-0.69)$ \\
Heart rate & $\geq 80$ beats per minute & 77 & 49 & $0.67(0.58-0.73)$ \\
FEV $1 \%$ ) predicted & $\leq 40 \%$ & 79 & 55 & $0.72(0.65-0.79)$ \\
CAT-score & $\geq 20$ & 56 & 79 & $0.69(0.61-0.77)$ \\
SF-6D V1-Score & $\leq 0.7$ & 82 & 43 & $0.65(0.58-0.73)$ \\
MMRC stage & $\geq 2$ & 75 & 51 & $0.67(0.60-0.74)$ \\
Number of COPD medications & $\geq 3$ & 88 & 61 & $0.72(0.66-0.79)$ \\
Platelet count & $\geq 270 \times 10^{9} / \mathrm{L}$ & 62 & 53 & $0.63(0.56-0.72)$ \\
IgG2 & $\leq 2.6 \mathrm{~g} / \mathrm{L}$ & 69 & $0.64(0.56-0.71)$ \\
\hline
\end{tabular}

COPD, chronic obstructive pulmonary disease; ROC, receiver operating characteristic; $A E C O P D$, acute exacerbation of chronic obstructive pulmonary disease; BMI, body mass index; AUC area under the curve.

moderate (Spearman's $r=0.40, p<0.001)$. In the 2 multivariable logistic regression models (the first model using all variables associated with previous frequent AECOPD - online suppl. Table 9 - and the second model using previous AECOPD in addition - online suppl. Table 10), previous AECOPD was not independently associated with frequent AECOPD during follow-up and did not change the performance of the model. The discriminatory power of the 2 multivariable ROC curves was the same (AUC 0.83) (shown in online suppl. Fig. 3).

\section{Discussion}

We investigated whether clinical parameters, COPD medication use, and biomarkers measured in an exacerbation-free interval in COPD patients were associated with AECOPD frequency and severity during the previous and the following year. We found that $\mathrm{FEV}_{1} \%$ predicted, number of COPD medications, platelet count, and IgG2 levels were independently associated with AECOPD frequency during the previous year. Heart rate was inde- 
pendently associated with severe AECOPD (requiring hospitalization or emergency room visit). The consistency between retrospective and prospective data was further assessed by multivariable ROC analysis. The model derived using baseline measurements and AECOPD data from the previous year showed fair power in predicting whether a patient experienced infrequent ( 0 or 1$)$ or frequent $(>1)$ AECOPD events during the following year.

\section{Independent Association of Clinical Parameters and Medication Use with AECOPD}

$\mathrm{FEV}_{1} \%$ predicted is a significant predictor for AECO$\mathrm{PD}$ frequency $[5,6]$ and severity [6]. Airway obstruction $\left(\mathrm{FEV}_{1} \%\right.$ predicted or $\mathrm{FEV}_{1}$ or GOLD stage) was the most common predictor in a meta-analysis comparing $27 \mathrm{AE}-$ COPD predictor models [21]. Possible reasons for elevated resting heart rates in COPD patients are enhanced sympathetic tone due autonomic dysfunction [22] or the systemic adrenergic effect of inhaled beta agonists [23]. However, in our study, we found that heart rate was associated with severe AECOPD independently of COPD medication use (online suppl. Table 5). A recent systematic review and meta-analysis showed that the elevated heart rate was associated with AECOPD frequency and severity, and death in COPD patients, possibly due to concomitant cardiovascular disease [24]. A further systematic review and meta-analysis showed that comorbidities including depression, alcohol use, renal, or heart failure, were associated with 30-day all-cause hospital readmission in COPD patients [25]. In the current study, we did not find an independent association between biomarkers for renal or heart failure with severe AECOPD events (requiring hospital admission - online suppl. Table 5). However, we found that NT-pro-BNP was associated with death from any cause during the year after baseline.

The number of COPD medication and the use of combined LAMA/LABA/ICS medication was higher in patients with more frequent and severe AECOPD. The likely explanation for higher use of LAMA/LABA/ICS therapy in those patients is the stepwise COPD therapy management leading to prescription of additional COPD medications in more severely ill patients.

\section{Association between Biomarkers and AECOPD}

Of the investigated biomarkers, platelet count and IgG2 were independently associated with previous AECOPD frequency. An association between thrombocytosis and AECOPD severity and mortality has also been shown in another study [26]. Hypogammaglobulinemia

Clinical Parameters and Biomarkers in the Swiss Multicenter COPD Cohort is associated with increased AECOPD frequency and severity, and higher GOLD stage [10,27]. Immunoglobulin replacement therapy or prophylactic antibiotics in COPD patients with hypogammaglobulinemia resulted in lower AECOPD frequency and severity and in reduced corticosteroid and antibiotic use [28]. Furthermore, COPD patients treated with corticosteroids have significantly lower IgG, but not IgG2 levels compared to untreated patients [27]. We therefore assessed the direct impact of ICS treatment on IgG and IgG2 levels and observed significantly lower IgG2 levels in patients taking ICS. The association between IgG2 levels and AECOPD frequency was corrected for COPD medication, including ICS use, suggesting that low IgG2 levels have an effect on AECOPD on their own (Table 4). Further, IgG2 levels were associated with AECOPD independent of patient's $\mathrm{FEV}_{1} \%$ predicted or GOLD stage. A recent study showed that hypogammaglobulinemia (IgG-levels below $6 \mathrm{~g} / \mathrm{L}$ ) is more common in patients with higher GOLD or MMRC score [29]. Moreover - in line with our findings - hypogammaglobulinemia remained an independent risk factor for AECOPD after correction for $\mathrm{FEV}_{1} \%$ predicted and was associated with reduced survival. Specifically, low IgG2 levels were associated with severe AECOPD in the year before measurement [29].

MBL levels were not significantly associated with AECOPD frequency or severity. This finding is in accordance with the results from previous studies $[12,30]$. Three of 4 patients with the rare IFN-lambda rs8099917GG genotype [31] had severe AECOPD in the year before baseline, giving a high OR for possession of this genotype and severe AECOPD. Investigations whether patients with GG-subtype have lower circulating INF-lambda concentrations are inconsistent $[17,31]$. The exact mechanism by which the GG genotype influences the expression of IFN-lambda 3 and hence antiviral response requires further research $[15,16]$. Our findings are in line with a previous study which found that AECOPD severity and outcome are both significantly associated with the rs8099917GG genotype [18].

\section{Baseline Data and Prediction of Frequent AECOPD \\ Events during Follow-Up}

Our ROC model derived from retrospective data showed AUC values close to 0.8 for predicting $>1$ AECO$\mathrm{PD}$ event in both the previous and in the following year, indicating that the parameters, which were obtained at a single point in time, are robust predictor variables. Despite being independently associated with AECOPD frequency, biomarkers (IgG2 and platelet count) did not im- 
prove the discriminatory power of the ROC model (Table 5, shown in Fig. 2). In this study, we therefore did not find a major role for the biomarkers measured during an exacerbation-free period in the development of AECOPD.

By way of comparison, a previous model including biomarkers (hemoglobin count and CRP levels) and clinical parameters (use of long-term oxygen therapy and CAT-score) predicted AECOPD with an AUC of 0.75 [32]. Another multivariate model also included the total number of COPD medication among other variables (daily use of COPD medication, number of AECOPD events during the previous year, $\mathrm{FEV}_{1} \%$ per forced vital capacity ratio, and female sex) and showed an AUC of 0.67 [4]. Other clinical variables included in our model, such as $\mathrm{BMI}, \mathrm{FEV}_{1} \%$ predicted, and MMRC, are used in the CODEXS and BODEX multicomponent scores [7]. ROC curve assessment for predicting frequent $A E C O P D$ showed an AUC of 0.82 using the CODEXS score and an AUC of 0.79 using the BODEX score [7].

\section{Limitations}

Despite being able to develop a model for predicting AECOPD frequency that had fair discriminatory power and included blood-based biomarkers, our study also has a number of limitations. These limitations mainly involve missing data and lack of follow-up data in one-third of all the patients included at baseline. A further limitation is the absence of an independent validation dataset. However, the ROC analysis that included the number of AECOPD events in the past year in addition to the other variables which predicted AECOPD performed the same after inclusion of number of AECOPD events in the past year, arguing against a significant confounder effect due to intraindividual correlation.

The genotyping of the IFN-lambda SNP could not be conducted in all patients because of low DNA quantity in the samples. The exacerbation frequency was self-reported; had formal exacerbation diary cards been used, they might have identified additional exacerbations. Another limitation is the small sample size, particularly with respect to subgroup analyses such as the association between oral corticosteroid use and immunoglobulin levels. In this study, we did not assess COPD associated with biomass smoke exposure so the findings are not generalizable to this group. There were no data about the presumed underlying cause of AECOPD, airway infiltration with inflammatory cells, or whether antibiotic treatment was given or not.

Despite these limitations, our findings in this wellcharacterized, real-life cohort of COPD patients are sim- ilar to those of previous studies from other countries, indicating generalizability. Furthermore, our study shows that the assessment of clinical and biological markers at one single point in time could be used to predict future exacerbations and therefore to aid patient management. These parameters could help physicians to decide whether a patient should be monitored more closely - for example with more frequent clinical assessments - and whether treatment escalations should be considered, even when FEV1\% predicted remains stable. Further studies are required to confirm our findings and their implications for everyday clinical practice.

\section{Conclusion}

In this cohort of COPD patients assessed at a single point in time, the frequency of AECOPD in the year before and the year after correlated with clinical and biomarker variables. However, the addition of biomarker variables to a predictive model using clinical parameters did not improve performance in discriminating between frequent and infrequent AECOPD.

\section{Acknowledgments}

There were no substantive contributions to this research from nonauthor individuals or an organization.

\section{Statement of Ethics}

The study was conducted in accordance with the Declaration of Helsinki of the World Medical Association. The study has been registered at Clinical Trails.gov NCT01527773. Ethics committees of all participating centers approved the study protocol (lead center Zurich KEK-ZH-NR: 2011-0106). All patients provided written informed consent.

\section{Conflict of Interest Statement}

C. Clarenbach received advisory fees from Roche, Novartis, Boehringer, GSK, Astra Zeneca, Sanofi, Vifor, and Mundipharma within the last 36 months, outside the submitted work. T. Dieterle is a board member of the Swiss Society of Hypertension. M. Trendelenburg is a recipient of a project grant of the Swiss National Science Foundation (Grant No. 310030_172956/1) and has research collaborations with Roche, Novartis, and Idorsia (all Switzerland), outside the submitted work. M. Trendelenburg reports presentations at local teaching sessions for Rheumatologists and at the Swiss Allergy and Immunology Update. M. Trendelenburg also reports support for the attendance of the European Lupus Ex- 
pert Forum. M. Trendelenburg is the president of the Association of the Swiss SLE Cohort Study. M. Kohler receives unrestricted grants from Lunge Zurich and reports consulting fees from GSK and participation on advisory boards of GSK, Novartis, Boehringer Ingelheim, Astra Zeneca, and Bayer, outside the submitted work. M. Kohler is a board member of the Swiss Society of Pneumology. M. Kohler is also a board member and cofounder of Deep Breath Intelligence, a company that provides services in the field of breath analysis. J.D. Leuppi is supported by grants from the Swiss National Science Foundation (SNF 160072 and 185592) as well as by Swiss Personalised Health Network (SPHN 2018DR108). J.D. Leuppi has also received unrestricted grants from AstraZeneca AG Switzerland, Boehringer Ingelheim GmbH Switzerland, GSK AG Switzerland, and Novartis AG Switzerland. All other authors have nothing to disclose.

\section{Funding Sources}

The study was supported by Lunge Zürich, Lungenliga St. Gallen, Lungenliga Thurgau, Lungenliga Glarus, Lungenliga Aargau, Lungenliga Beider Basel, the Gottfried und Julia BangerterRhyner-Stiftung, and the Freiwillige Akademische Gesellschaft Basel.

\section{Author Contributions}

S.T. Hübner and S. Henny contributed equally to data analyses and writing of this manuscript. S. Giezendanner contributed to the statistical analyses. T. Brack, M.H. Brutsche, P.N. Chhajed, C.F. Clarenbach, T. Dieterle, M. Frey, S. Irani, N.A. Sievi, R. Thurnheer, M. Trendelenburg, M. Kohler, and J.D. Leuppi were responsible for designing and carrying out the study in each of the 7 participating Swiss teaching hospitals. A. Egli, I. Heijnen, and M. Trendelenburg performed laboratory analyses and contributed to the evaluation of the biomarkers tested in the current study. A.B. LeuppiTaegtmeyer and J.D. Leuppi contributed to writing and discussion of the manuscript.

\section{Data Availability Statement}

All data generated or analyzed during this study are included in this article and its online supplementary material files. Further inquiries can be directed to the corresponding author.

\section{References}

1 GBD 2017 Causes of Death Collaborators. Global, regional, and national age-sex-specific mortality for 282 causes of death in 195 countries and territories, 1980-2017: a systematic analysis for the Global Burden of Disease Study 2017. Lancet. 2018;392:1736-88.

2 Jochmann A, Scherr A, Jochmann DC, Miedinger D, Török SS, Chhajed PN, et al. Impact of adherence to the GOLD guidelines on symptom prevalence, lung function decline and exacerbation rate in the Swiss COPD cohort. Swiss Med Wkly. 2012;142: w13567.

3 Burge S, Wedzicha JA. COPD exacerbations: definitions and classifications. Eur Respir J Suppl. 2003;41:46s-53s.

4 Make BJ, Eriksson G, Calverley PM, Jenkins CR, Postma DS, Peterson S, et al. A score to predict short-term risk of COPD exacerbations (SCOPEX). Int J Chron Obstruct Pulmon Dis. 2015;10:201-9.

5 Urwyler P, Abu Hussein N, Bridevaux PO, Chhajed PN, Geiser T, Grendelmeier P, et al. Predictive factors for exacerbation and re-exacerbation in chronic obstructive pulmonary disease: an extension of the Cox model to analyze data from the Swiss COPD cohort. Multidiscip Respir Med. 2019;14:7.

6 Hoogendoorn M, Feenstra TL, Boland M, Briggs AH, Borg S, Jansson SA, et al. Prediction models for exacerbations in different COPD patient populations: comparing results of five large data sources. Int J Chron Obstruct Pulmon Dis. 2017;12:3183-94.
7 Deng D, Zhou A, Chen P, Shuang Q. CODEXS: a new multidimensional index to better predict frequent COPD exacerbators with inclusion of depression score. Int J Chron Obstruct Pulmon Dis. 2020;15:249-59.

8 Wedzicha JA, Brill SE, Allinson JP, Donaldson GC. Mechanisms and impact of the frequent exacerbator phenotype in chronic obstructive pulmonary disease. BMC Med. 2013;11:181.

9 Hurst JR, Vestbo J, Anzueto A, Locantore N, Müllerova H, Tal-Singer R, et al. Susceptibility to exacerbation in chronic obstructive pulmonary disease. N Engl J Med. 2010;363(12): 1128-38.

10 Leitao Filho FS, Ra SW, Mattman A, Schellenberg RS, Criner GJ, Woodruff PG, et al. Serum IgG subclass levels and risk of exacerbations and hospitalizations in patients with COPD. Respir Res. 2018;19(1):30.

11 Putcha N, Paul GG, Azar A, Wise RA, O’Neal WK, Dransfield MT, et al. Lower serum IgA is associated with COPD exacerbation risk in SPIROMICS. PLoS One. 2018;13(4): e0194924.

12 Lin CL, Siu LK, Lin JC, Liu CY, Chian CF, Lee $\mathrm{CN}$, et al. Mannose-binding lectin gene polymorphism contributes to recurrence of infective exacerbation in patients with COPD. Chest. 2011;139(1):43-51.

13 Jewell NA, Cline T, Mertz SE, Smirnov SV, Flaño E, Schindler C, et al. Lambda interferon is the predominant interferon induced by influenza A virus infection in vivo. J Virol. 2010; 84(21):11515-22.
14 Khaitov MR, Laza-Stanca V, Edwards MR, Walton RP, Rohde G, Contoli M, et al. Respiratory virus induction of alpha-, beta- and lambda-interferons in bronchial epithelial cells and peripheral blood mononuclear cells. Allergy. 2009;64(3):375-86.

15 Egli A, Santer DM, O'Shea D, Tyrrell DL, Houghton M. The impact of the interferonlambda family on the innate and adaptive immune response to viral infections. Emerg Microbes Infect. 2014;3(7):e51.

16 Syedbasha M, Egli A. Interferon lambda: modulating immunity in infectious diseases. Front Immunol. 2017;8:119.

17 Alborzi A, Hashempour T, Moayedi J, Musavi Z, Pouladfar G, Merat S. Role of serum level and genetic variation of IL-28B in interferon responsiveness and advanced liver disease in chronic hepatitis $\mathrm{C}$ patients. Med Microbiol Immunol. 2017;206(2):165-74.

18 Egli A, Mandal J, Schumann DM, Roth M, Thomas B, Lorne Tyrrell D, et al. IFN $\Lambda 3 / 4$ locus polymorphisms and IFN $\Lambda 3$ circulating levels are associated with COPD severity and outcomes. BMC Pulm Med. 2018;18(1):51.

19 Schonmann M, Sievi NA, Clarenbach CF, Brack T, Brutsche M, Frey M, et al. Physical activity and the frequency of acute exacerbations in patients with chronic obstructive pulmonary disease. Lung. 2015;193(1):63-70.

20 Ekelund S. ROC curves-what are they and how are they used? Point Care. 2012;11(1): 16-21. 
21 Guerra B, Gaveikaite V, Bianchi C, Puhan MA. Prediction models for exacerbations in patients with COPD. Eur Respir Rev. 2017; 26(143):160061.

22 van Gestel AJ, Steier J. Autonomic dysfunction in patients with chronic obstructive pulmonary disease (COPD). J Thorac Dis. 2010; 2(4):215-22.

23 Salpeter SR, Buckley NS, Ormiston TM, Salpeter EE. Meta-analysis: effect of long-acting beta-agonists on severe asthma exacerbations and asthma-related deaths. Ann Intern Med. 2006;144(12):904-12.

24 Fermont JM, Masconi KL, Jensen MT, Ferrari R, Di Lorenzo VAP, Marott JM, et al. Biomarkers and clinical outcomes in COPD: a systematic review and meta-analysis. Thorax. 2019;74(5):439-46.

25 Alqahtani JS, Njoku CM, Bereznicki B, Wimmer BC, Peterson GM, Kinsman L, et al. Risk factors for all-cause hospital readmission following exacerbation of COPD: a systematic review and meta-analysis. Eur Respir Rev. 2020;29(156):190166.
26 Harrison MT, Short P, Williamson PA, Singanayagam A, Chalmers JD, Schembri S. Thrombocytosis is associated with increased short and long term mortality after exacerbation of chronic obstructive pulmonary disease: a role for antiplatelet therapy? Thorax. 2014;69(7):609-15.

27 O'Keeffe S, Gzel A, Drury R, Cullina M, Greally J, Finnegan P. Immunoglobulin G subclasses and spirometry in patients with chronic obstructive pulmonary disease. Eur Respir J. 1991;4(8):932-6.

28 McCullagh BN, Comellas AP, Ballas ZK, Newell JD Jr, Zimmerman MB, Azar AE. Antibody deficiency in patients with frequent exacerbations of Chronic Obstructive Pulmonary Disease (COPD). PLoS One. 2017;12(2): e0172437.
29 Holm AM, Andreassen SL, Christensen VL, Kongerud J, Almås $\emptyset$, Auråen $\mathrm{H}$, et al. Hypogammaglobulinemia and risk of exacerbation and mortality in patients with COPD. Int J Chron Obstruct Pulmon Dis. 2020;15:799807.

30 Mandal J, Malla B, Steffensen R, Costa L, Egli A, Trendelenburg M, et al. Mannose-binding lectin protein and its association to clinical outcomes in COPD: a longitudinal study. Respir Res. 2015;16:150.

31 Egli A, Santer DM, O'Shea D, Barakat K, Syedbasha M, Vollmer M, et al. IL-28B is a key regulator of $\mathrm{B}$ - and $\mathrm{T}$-cell vaccine responses against influenza. PLoS Pathog. 2014;10(12): e1004556.

32 Garcia-Rivero JL, Esquinas C, Barrecheguren $\mathrm{M}$, Bonnin-Vilaplana $\mathrm{M}$, Garcia-Sidro $\mathrm{P}$, Herrejon A, et al. Risk factors of poor outcomes after admission for a COPD exacerbation: multivariate logistic predictive models. COPD. 2017;14(2):164-9. 